\title{
Electronic nature of the enhanced conductivity in YSZ-STO multilayers deposited by PLD
}

Andrea Cavallaro ${ }^{1}$, Mónica Burriel ${ }^{2}$, Jaume Roqueta ${ }^{1}$, Alexandra Apostolidis ${ }^{1}$, Alessandro Bernardi ${ }^{1}$, Albert Tarancón ${ }^{3}$, Srini Rajagopalan ${ }^{4}$, Stuart N. Cook ${ }^{2}$, Hamish L. Fraser ${ }^{4}$, John A. Kilner $^{2}$, David W. McComb ${ }^{2}$ and José Santiso,

${ }^{I}$ Centro de Investigación en Nanociencia y Nanotecnologia, CIN2 (CSIC-ICN). Campus UAB. 08193 Bellaterra, Spain

${ }^{2}$ Dept. of Materials, Imperial College London, London SW7 2AZ, UK,

${ }^{3}$ Institute for Microelectronics in Barcelona IMB-CNM (CSIC) Campus UAB. 08193 Bellaterra, Spain

${ }^{4}$ Dept of Materials Science and Engineering, The Ohio State University, Columbus, Ohio, USA.

*corresponding author, e-mail: jsantiso@cin2.es

Keywords: charge transport; thin films; interfaces; epitaxial heterostructures; ionic conductivity;

PLD 


\begin{abstract}
The search for new strategies to enhance the oxide ionic conductivity in oxide materials is a very active field of research. These materials are needed for application in a new generation of more efficient and durable solid state electrochemical devices such as reduced-temperature Solid Oxide Fuel Cells (SOFC's). Recently published results have claimed that sputtered yttria stabilised zirconia (YSZ)/ $\mathrm{SrTiO}_{3}$ (STO) heterostructures show enhanced oxygen ion conductivity, by about eight orders of magnitude, with respect to that expected for YSZ and STO bulk values. The YSZ/STO heterostructures obtained in this work from Pulsed Laser Deposited (PLD) films, also show enhanced conductivity of a similar order. However, the fact that our structures show different relative orientations indicates that the conductivity enhancement may not be associated to a particular crystallographic arrangement at the interface. The combined characterisation of the conductivity dependence on oxygen partial pressure and direct oxygen diffusion by means of tracer experiments clearly demonstrate that the enhancement observed is related to the electronic rather than ionic conductivity.
\end{abstract}




\section{Introduction}

In the last few years there has been a major effort from the solid state community to find new strategies to enhance the ionic conductivity for electrochemical devices such as solid oxide fuel cells (SOFCs) $[1,2]$. Along with the quest for new materials there has been a substantial interest in improving the performance of existing ionic conducting materials through control of their structure at the nanoscale $[3,4]$. It has been shown that reducing the grain size of yttriastabilised zirconia (YSZ) [5] and $\mathrm{CeO}_{2}$ [6] in polycrystalline single phase material to a few nanometres significantly influences the conductivity. This effect was related to the variation of the charge carriers density, or their mobilities, indicating the importance of the interfaces in enhancing charge transport phenomena beyond trivial size effects of increasing surface-tovolume ratio in nanocrystalline material. Epitaxial heterostructures prepared by sequential deposition of $\mathrm{CaF}_{2} / \mathrm{BaF}_{2}$ have also shown enhancement of the $\mathrm{F}^{-}$ionic conductivity along the interfaces, by several orders of magnitude, upon reducing the thickness to a few nanometers $[7,8]$. This effect was attributed to the variation of the density of majority charge carriers in the space charge region generated at the interfaces between both materials. Some multilayered heterostructures based on fluorite-type ionic conductors have also been reported to show an increase in conductivity. This is the case in Gd-doped $\mathrm{CeO}_{2}$ and $\mathrm{ZrO}_{2}$ multilayers grown on $\mathrm{Al}_{2} \mathrm{O}_{3}(0001)$ by oxygen plasma-assisted molecular beam epitaxy (MBE) [9] where up to one order of magnitude increase in planar conductivity was observed. Similarly, $\mathrm{CeO}_{2} / \mathrm{Sm}$-doped $\mathrm{CeO}_{2}$ epitaxial multilayers have shown an enhanced conductivity of around one order of magnitude that was attributed to the oxygen ions [10]. In recent experimental studies by Janek $e t$ al [11-14] it has been reported that the strain in epitaxial multilayers of YSZ and several $\mathrm{Re}_{2} \mathrm{O}_{3}$ 
oxides $(\mathrm{Re}=\mathrm{Y}, \mathrm{Lu}, \mathrm{Sc})$ may significantly alter the mobility of the oxygen ions depending on the sign of the strain. Altogether these studies show clear evidence that charge carrier densities and/or mobilities can be manipulated by interface engineering and that artificial (meta) materials can show enhanced conductivity. The most outstanding recent example of this concerns YSZ/STO multilayers which are claimed to exhibit about eight orders of magnitude increase in ionic conductivity as a result of a particular interface structure that was induced in fully strained ultrathin $(\sim 1 \mathrm{~nm})$ YSZ layers [15]. These last results have been the subject of much debate in the solid state community focusing upon the ionic or electronic nature of the observed effect $[4,14,16,17]$. In this study YSZ/STO epitaxial heterostructures were prepared by pulsed laser deposition (PLD) in order to investigate the conductivity in this system. The particular microstructure of PLD films is reported and related it to the mass and charge transport properties with a particular emphasis in clarifying whether the observed enhancement in conductivity has an electronic or ionic nature.

\section{Experimental}

\subsection{Thin film deposition}

The PLD films were grown using a Compex Pro $201 \mathrm{KrF}$ excimer laser (248 nm) at $1 \mathrm{~Hz}$ pulse repetition at a laser fluence of around $2 \mathrm{~J} / \mathrm{cm}^{2}\left(80 \mathrm{~mJ}\right.$ in $4 \mathrm{~mm}^{2}$ spot size $)$. We sequentially ablated YSZ $\left(8 \mathrm{~mol} \% \quad \mathrm{Y}_{2} \mathrm{O}_{3}: \mathrm{ZrO}_{2}\right)$ and $\mathrm{SrTiO}_{3}$ (STO) dense targets in a $4.7 \mathrm{~Pa}$ atmosphere of pure $\mathrm{O}_{2}$. Substrates were commercial single crystals of STO(001) (Crystal, GmbH). Substrate temperatures were set to $900{ }^{\circ} \mathrm{C}$. Target to substrate distance was fixed at about $50 \mathrm{~mm}$. Prior to YSZ deposition in all experiments we deposited a thin $10 \mathrm{~nm}$ STO homoepitaxial buffer layer. 
This is a general procedure in order to avoid the possible defects arising from a mixed Sr-O, Ti$\mathrm{O}_{2}$ surface termination on as-received STO substrates [18]. STO/YSZ bilayers, STO/YSZ/STO trilayers and [STO/YSZ]x10 multilayers were obtained with different YSZ thickness from 1 to 5 $\mathrm{nm}$, whereas STO thickness of individual layers was kept to $10 \mathrm{~nm}$, in order to facilitate comparison the previously reported results [15]. In most of the experiments the growth of the layers was characterised in-situ by reflection high energy electron diffraction (RHEED) by using a electron gun (RDEC Co. Ltd) operated at $30 \mathrm{kV}$ and $50 \mu \mathrm{A}$. The RHEED patterns were collected at a minimum $12 \mathrm{~ms}$ acquisition time by a CCD camera (kSA400, K-Space Associates, Inc.). After deposition the films were cooled down under the same $p \mathrm{O}_{2}$ and subsequently analysed by X-ray diffraction (XRD) and atomic force microscopy (AFM). The thickness of the YSZ layers was estimated as proportional to the number of pulses after a previous calibration on thicker YSZ films measured by X-ray reflectometry, whereas for STO the thickness was extracted directly from the observed RHEED oscillations.

\subsection{TEM specimen preparation}

TEM cross-sections from the trilayers and multilayers films were prepared using a focussed ion beam (FIB) system (FEI Helios nanolab). A $50 \mathrm{~nm}$ thick coating of Au was evaporated onto the sample before FIB milling to protect the region of interest and prevent charging. Coarse milling was done at $30 \mathrm{kV}$ and then samples were thinned to approximately 50

$\mathrm{nm}$ thickness at $2 \mathrm{kV}$. The amorphous damage layer was removed by localised Ar-ion milling (Fischione Nanomill) at 800 V. Samples were examined in an aberration corrected scanning transmission electron microscope (STEM, FEI Titan) operated at $300 \mathrm{kV}$ using a high angle annular dark field (HAADF) detector. The microscope was fitted with electron energy-loss 
spectroscopy (EELS, Gatan Tridiem) and energy dispersive X-ray (EDX) spectroscopy (Oxford Instruments) facilities. Convergence and collection angles of $17 \mathrm{mrad}$ and $34 \mathrm{mrad}$, respectively, were used for STEM-EELS. A range of energy dispersions and acquisition times were used.

\subsection{Electrical characteristation}

In order to analyse the conductance of the films the in-plane AC impedance (Solartron 1260 and Agilent E4980 LCR) was measured between two Ag parallel contacts deposited onto the film surface. Open circuit capacitances of instrumental setup were measured to be of about $0.1 \mathrm{pF}$. Single $10 \mathrm{~nm} \mathrm{STO/STO}(001)$ and $5 \mathrm{~nm} \mathrm{YSZ} / \mathrm{Al}_{2} \mathrm{O}_{3}(1-102)$ thin films were also deposited by PLD for comparing AC impedance. The samples were measured upon several temperature cycles from RT to $500{ }^{\circ} \mathrm{C}$ under pure $\mathrm{O}_{2}$ and air atmosphere in order to assess heterostructure stability. Finally the conductance of the samples was measured under different $\mathrm{pO}_{2}$ by mixing $\mathrm{O}_{2}$ and Ar gas flows.

\subsection{Isotope exchange depth profiling}

The oxygen Isotope Exchange Depth Profile (IEDP) technique, was used to measure the diffusivity of the oxide ions within the PLD layers in order to unambiguously determine the nature of the charge carrier giving rise to the enhanced conductivity. An STO capping layer of approximately $145 \mathrm{~nm}$ was deposited by PLD (under the same deposition conditions) on top of the STO/YSZ/STO trilayers and [STO/YSZ]x10 multilayers films (with a YSZ thickness of 1 $\mathrm{nm})$ to prevent oxygen exchange from the top surface of the multilayer. The samples were then either cleaved or a trench was opened with a scalpel from the top surface, to expose the edges of 
the multilayer film to the ${ }^{18} \mathrm{O}$ enriched gas. Since we were mainly interested in the oxygen diffusion process, and in order to ensure that the oxygen surface exchange was not the limiting process (preventing the oxygen isotope from exchanging with the sample), a set of samples were sputtered with a Ag layer directly onto the cleaved edge, to enhance the surface exchange coefficient of the lateral exposed surface [19]. Prior to the isotopic exchange annealing, asprepared samples were placed in a silica tube and evacuated to $<9 \times 10^{-7}$ mbar by a turbomolecular pump backed by a rotary pump. The furnace was moved to heat the samples and these were then subjected to a pre-annealing in pure oxygen (research grade 99.9995\%) of natural isotopic abundance for a period of time approximately one order of magnitude greater than the ${ }^{18} \mathrm{O}$ tracer annealing time and at the same temperature as the subsequent exchange. The samples were then cooled to room temperature, the natural oxygen gas was removed and an ${ }^{18} \mathrm{O}$ enriched gas $(34.0 \%)$ was introduced. The samples were then reheated, by rolling the preheated furnace over the sample holder. A constant temperature (of 450 or $500{ }^{\circ} \mathrm{C}$ ) was maintained for 2 $\mathrm{h}$, and finally the samples were quenched by rolling the furnace off the sample holder. Once the samples had cooled the labeled oxygen was cryogenically recovered using molecular sieve sorption pumps. The ${ }^{18} \mathrm{O}$ exposure time and temperature of the exchange experiments were chosen in order to ensure that diffusion length within the YSZ layers (provided the oxygen diffusivity showed YSZ bulk values) would be within the range of $200-400 \mu \mathrm{m}$, as required for an optimal lateral resolution of the SIMS linescan.

Depth profiles of the exchanged samples were measured by secondary ion mass spectrometry (SIMS) using an Atomika 6500 quadrupole-based analyzer, sputtering with an argon primary ion beam at $500 \mathrm{eV}$ and an angle of incidence of $45^{\circ}$. To follow the isotopic distribution, the negative ions ${ }^{18} \mathrm{O}^{-},{ }^{16} \mathrm{O}^{-},{ }^{89} \mathrm{Y}^{16} \mathrm{O}^{-}$and ${ }^{90} \mathrm{Zr}^{16} \mathrm{O}^{-}$were monitored during the depth 
profile. To follow the isotopic distribution from the lateral exposed side longitudinal concentration profiles were also measured. This was achieved in two different ways: by using the line scan mode and by using the checkerboard mode in the Atomika SIMS system. Line scan mode describes the acquisition of the SIMS data from straight line of points (255 data points) along the centre of a square crater. Checkerboard mode provides a compositional map of the area in the centre of the crater by dividing it into a 16x16 array of squares ("checkerboard") and analysing the composition of each square individually. Both modes provide fast multipoint measurement of the composition over an extended area of the crater without substantially increase the acquisition time, and therefore increasing the depth resolution during a continuous etching experiment. Subsequently, the depth of the etched craters was measured using an optical microscope based interferometer, ZYGO Corp. NewView 200.

\section{Results and discussion}

\subsection{STO buffer layer}

The quality of the bottom STO layer was characterised before growth of the multilayered heterostructures. Figure 1 shows the RHEED pattern after PLD deposition of the bottom STO layer onto as-received $\mathrm{STO}(001)$ substrates (left) along with the intensity oscillations of the reflected beam (right) observed during the first 60 laser pulses. As expected for homoepitaxial growth under these conditions, the RHEED pattern did not significantly change during deposition and was almost identical to that recorded from the STO substrate. The intensity of the RHEED pattern is mainly related to the surface roughness on the atomic scale, so the observation of RHEED oscillations is generally associated to a high quality 2D layer-by-layer 
growth. The difference between two consecutive maxima corresponds to a single monolayer of the growing material, which for STO corresponds to a perovskite unit cell of about $0.3905 \mathrm{~nm}$. In the figure the oscillations of the overall intensity are clearly visible at least for the first six monolayers. The smaller jumps in the intensity correspond to each laser pulse at $1 \mathrm{~Hz}$ frequency. The main oscillation period takes place every 5 pulses, therefore the STO film growth rate is about $0.08 \mathrm{~nm} /$ pulse.

\subsection{STO/YSZ bilayers and STO/YSZ/STO trilayers}

\subsubsection{YSZ thickness dependence}

Figure 2 shows AFM images of the top STO surface morphology along with final RHEED patterns of epitaxial STO/YSZ/STO trilayers with different YSZ thickness from $1 \mathrm{~nm}$ to $5 \mathrm{~nm}(\mathrm{STO}$ thickness $=10 \mathrm{~nm})$. The morphology of the trilayer with $1 \mathrm{~nm} \mathrm{YSZ}$ reveals a stepped surface with height differences of about $0.4 \mathrm{~nm}$ corresponding to the STO unit cell length. Some holes about 20-30 nm in diameter were also observed. As the thickness of the YSZ layer is increased from $1 \mathrm{~nm}$ to $5 \mathrm{~nm}$ the surface becomes progressively rougher (rms values of $0.4 \mathrm{~nm}$ to $5 \mathrm{~nm}$, respectively) with an apparent grain size of about 50-100 nm. The corresponding RHEED patterns for [100] zone axis after the STO top layer deposition are also shown in Fig. 2 (right). The film with 1nm YSZ (Fig. 2.top) shows a streaky pattern along with defined spots on the zero order Laue zone indicating a flat surface similar to that of the STO substrate and buffer layer. Although the film with $2.5 \mathrm{~nm}$ YSZ still shows a streaky RHEED pattern, more defined spots typical for a transmission pattern are also observed. This is much clearer in the trilayer with $3.75 \mathrm{~nm}$ thickness, and is consistent with the progressive island morphology observed by AFM. However, in both cases the pattern still preserves the same crystal orientation of the 
underlying STO buffer layer and substrate. Trilayers with thicker YSZ films show progressive misorientation of the top surface STO domains, even showing powder-like rings for $5 \mathrm{~nm}$ YSZ (Fig 2. bottom). A large decay in the RHEED overall pattern intensity was observed at the start of the YSZ deposition in real-time acquisitions, which made indexing of YSZ patterns at the same conditions as the STO patterns more challenging. No RHEED oscillations were observed during YSZ layer growth. However, RHEED pattern intensity recovers very quickly after subsequent deposition of the top STO layer, and RHEED oscillations are again observed with the same amplitude indicating the full reconstruction of the 2D layer-by-layer growth, at least for the YSZ $1 \mathrm{~nm}$ nominal thickness.

\subsubsection{YSZ Orientation relationship}

In order to analyse in more detail the crystallographic orientation of the YSZ layers overexposed RHEED patterns were collected during the YSZ deposition step. The RHEED pattern in Fig. 3 (a) was taken after $2.5 \mathrm{~nm}$ YSZ in trilayer deposition at [100] STO zone axis. The spotted pattern indicates a 3D growth of YSZ whose orientation could not be indexed as corresponding to the expected cube-on-cube epitaxy, i.e.: $\mathrm{YSZ}(001) / / \mathrm{STO}(001)$ and [110]YSZ//[100]STO. Instead, the pattern seemed to match very well to an overlap between coexisting $15^{\circ}$-tilted (111)YSZ-oriented domains, with tilt axis parallel to $<100>$ STO in-plane axes (Fig. 3(b)). A simulated pattern of such a combination of oriented YSZ domains is superimposed with the RHEED experimental pattern shows a perfect match. XRD patterns of heterostructures with very thin YSZ layer $(1 \mathrm{~nm})$ only revealed the presence of STO reflections without any features from the YSZ structure, probably because of the small YSZ volume. However, STO $(10 \mathrm{~nm}) / \mathrm{YSZ}$ bilayers with YSZ thickness of about $5 \mathrm{~nm}$ reveal XRD peaks at $2 \theta$ 
angles corresponding to YSZ bulk material. XRD pole figure measurements of 111 and 200 YSZ reflections $\left(2 \theta=30.0^{\circ}\right.$ and $34.8^{\circ}$, respectively) are shown in Fig. 3(c). They indicate the coexistence of four YSZ domains with an orientation close to 111 direction, although slightly tilted from the vertical direction about $15^{\circ}$ towards the main 100 substrate directions which is fully consistent with the orientations extracted from the RHEED patterns.

\section{3. $(\text { STO/YSZ) })_{x 10}$ multilayers}

\subsubsection{Surface morphology and XRD characterisation}

Surface morphology of top STO layer in $[\mathrm{STO}(10 \mathrm{~nm}) / \mathrm{YSZ}(1 \mathrm{~nm})] \times 10$ multilayer is shown in Fig. 4(a). Although it does not show unit cell steps the surface morphology is very smooth $(\mathrm{rms}<1 \mathrm{~nm})$ indicating a high quality multilayer growth. Figure $4(b)$ shows the highresolution XRD pattern around the 002 Bragg reflection of the $[\mathrm{STO} / \mathrm{YSZ}(1 \mathrm{~nm})] \mathrm{x} 10$ multilayer. Along with intense substrate 002 reflection the graph shows a broader main peak at $2 \theta=46.16^{\circ}$, corresponding to an average multilayer cell parameter of $0.393 \mathrm{~nm}$, and up to four satellite peaks at both sides of the main peak, which is an indication of the high crystal coherence of the multilayer heterostructure. This is in good agreement with the simulated pattern for perfectly flat interfaces shown in Fig 4(b). The multilayer periodicity associated to the satellite separation corresponds to $\Lambda=9.6 \mathrm{~nm}$ which is slightly less than the expected $11 \mathrm{~nm}(10 \mathrm{~nm}$ STO $+1 \mathrm{~nm}$ YSZ). The X-ray reflectivity of the same multilayer also reveals a multilayer periodicity of about $10 \mathrm{~nm}$, but shows a poor oscillation amplitude in comparison to a simulated curve with flat interfaces. (Fig 4(c)) This is an indication that either, the interface roughness is considerably 
larger, or, the contrast between individual layers is lower than that expected for YSZ and STO as result of the formation of other phases.

\subsubsection{Multilayer cross sections microstructure}

Figure $5(a$ and $b)$ show HAADF-STEM images from a cross section of a STO(10 $\mathrm{nm}) / \mathrm{YSZ}(1 \mathrm{~nm}) / \mathrm{STO}(10 \mathrm{~nm})$ trilayer prepared by FIB milling. The large islands at the top of Fig. 5(a) that exhibit bright contrast are associated with the Au protective coating. It is difficult to identify any contrast associated with the interface between the STO substrate and the 10nm STO buffer layer, and the high resolution image establishes coherent homoepitaxial STO growth has occurred. (Fig. 5(b)) It is immediately evident that the YSZ layer is non-continuous: it consists of a series of YSZ islands as confirmed by EELS and EDX analysis. The YSZ islands range from 5-15 $\mathrm{nm}$ in width and 3-4 $\mathrm{nm}$ in height. Utilising the small depth of focus of an aberration-corrected electron probe it was established that some of the islands are 30-40 nm long suggesting a rod-like structure. The islands exhibit a range of different tilt and orientation relationships to the STO substrate. The subsequent deposition of STO results in coherent growth of STO on both the STO buffer layer and above the YSZ islands. However, this STO layer is not uniformly thick. It appears that it grows as a series of "hut-like" structures that are equally spaced, have a base that is $\sim 60 \mathrm{~nm}$ and a top that is $\sim 40 \mathrm{~nm}$ with $\{110\}$ facets. This faceted growth means that some YSZ islands have no STO layer above them and effectively leaves a series of depressions or holes on the top surface which may correlate with those observed in the AFM image in Fig. 1. These depressions act as preferential nucleation sites for Au (confirmed by EDS analysis) and the facets seem to result in strongly columnar growth in the Au protective coating. 
A clearer insight to film microstructure is obtained from STEM-HAADF imaging of multilayer heterostructures obtained after sequential deposition (x10 periods) of $\mathrm{STO}(10 \mathrm{~nm})$ and YSZ(1 nm), and top covered by an additional $10 \mathrm{~nm}$ STO layer. A number of HAADF images from a cross-section of one of the multilayer films are shown in Fig. 5 ( $c$ to $f$ ). The intensity of the HAADF signal is proportional to $\mathrm{Z}^{\mathrm{n}}(\mathrm{n} \sim 2)$ where $\mathrm{Z}$ is atomic number averaged through the thickness of the sample. It is for this reason that the Au islands in Fig. 5(a) exhibit bright contrast. Correspondingly, in Figure 5(c) the bright islands are associated with YSZ, as it corresponds to a larger average $\mathrm{Z}$ compared with STO. While the multilayer structure is clearly apparent in Fig. 5(c), the YSZ layers are discontinuous and are typically about 3-4 nm in height rather than $1 \mathrm{~nm}$. It is clear that there is considerable intergrowth of YSZ and STO in the first three periods of the multilayer. In Fig. $5(d)$ the $2^{\text {nd }}, 3^{\text {rd }}$ and $4^{\text {th }}$ YSZ layers are shown at higher magnification. Although intergrowth of YSZ and STO is observed between the $2^{\text {nd }}$ and $3^{\text {rd }}$ YSZ layers, the lattice in these regions is entirely coherent with the STO lattice. Remarkably, the growth mode after the $3^{\text {rd }}$ YSZ layer alters and there is no further intergrowth in subsequent layers. This suggests that the facetted "hut-like" features observed in the trilayer sample, persist over approximately three periods in the multilayer structure before true layer-by-layer growth resumes. In the remaining layers YSZ islands exhibiting a range of morphologies are observed. At least two distinct orientation relationships are observed: $i)[001]_{\text {STO }} \|[001]_{\text {YSZ }}$ and (020) $)_{\text {STO }} \|$ $(2-20)_{\text {YsZ }}$ which corresponds to the expected epitaxial orientation as reported in [15]; ii) [001 $]_{\text {STO }}$ \| [1-10] $]_{\mathrm{YsZ}}$, and (110) $)_{\mathrm{STO}} \|(00-2)_{\mathrm{YSZ}}$ which is consistent with the $15^{\circ}$-tilted (111)YSZ domains observed by RHEED and XRD. In each of these relationships very large elastic strains ( 7\%) are required for lattice matching. An incoherent interface is expected along at least one direction which is clearly observed in the islands shown in Fig. 5(e). The chain-like structure shown in Fig. 5(f) is commonly observed and seems to correspond to either, between four and eight (002) 
atomic planes of tilted YSZ growing on (110) STO facets, or, to a perovskite with a composition clearly different to that of surrounding STO. The "halo" around one of the islands in Fig. 5(d) could be due to strain contrast from chain-like structures aligned along [1-10] YSZ.

\subsubsection{EELS composition analysis}

The chemical composition of the multilayer was analysed by EELS in different regions shown in Fig. 6(a). The characteristic energy-loss spectra in the energy range 1900-2500 eV from STO and YSZ show sharp ionisation edges superimposed on a decreasing background associated with transitions at lower energy-losses (Fig. 6(b)). Each of the transition elements in STO and YSZ exhibits two sharp peaks or "white lines" (Sr: $1972 \mathrm{eV} \mathrm{\&} 2041 \mathrm{eV}, \mathrm{Y}: 2115 \mathrm{eV} \&$ $2193 \mathrm{eV}, \mathrm{Zr}: 2261 \mathrm{eV} \& 2346 \mathrm{eV})$ which are associated with the $\mathrm{L}_{3^{-}}\left(2 \mathrm{p}_{3 / 2} \rightarrow \mathrm{d}\right)$ and $\mathrm{L}_{2^{-}}$ $\left(2 p_{1 / 2} \rightarrow d\right)$ transitions, respectively. Note that these values are approximately $40 \mathrm{eV}$ higher than literature values due to an absolute error in the energy calibration. For the $\mathrm{Sr}_{2,3}$-edge a power law function fitted to the background prior to the edge can be extrapolated and then subtracted from the spectrum. The integrated intensity of the $\mathrm{Sr}$ signal in an energy window $(\Delta E=50 \mathrm{eV})$ placed on the $\mathrm{Sr} \mathrm{L}_{2,3}$-edge (as it is depicted in the gray areas in Fig.6(b)) can then be plotted as a function of position in different regions to obtain a compositional profile (fig. 6(c)). The energy overlap of the edges makes the same procedure more difficult for the $\mathrm{Y}$ and $\mathrm{Zr}$ edges, particularly at the interfacial regions where $\mathrm{Sr}$ signal might coexist. In the energy region immediately prior to the $\mathrm{Zr}$ edge, the $\mathrm{Sr}$ signal has a shape that is close to a power law background, so it can be easily corrected and the $\mathrm{Zr}$ profile can thus be extracted with enough certainty. However, this procedure cannot be applied to the $\mathrm{Y}$ edge and consequently no $\mathrm{Y}$ profiles have been extracted. The use of multiple linear least squares (MLLS) fitting is being investigated to obtain this profile. In Fig. 6(c), profiles showing the $\mathrm{Sr}$ and $\mathrm{Zr}$ signals, as well as 
the intensity of the HAADF signal are presented. The profiles were acquired from a YSZ island (\#1), a STO region between islands (\#2), and a chain-like YSZ structure (\#3), as indicated in the image in Fig. 6(a). Each profile is $10 \mathrm{~nm}$ in length and was acquired using a probe size of $0.2 \mathrm{~nm}$ and a step size of $0.25 \mathrm{~nm}$. Profile \#1 shows that the islands contain $\mathrm{Zr}$, do not contain $\mathrm{Sr}$ and have relatively sharp interfaces with little evidence of inter-diffusion. Profile \#2 shows that the

$\mathrm{Sr}$ concentration in the region between the islands is unaltered relative to the bulk STO layers. Profile \#3 shows that the chain-like features are $\mathrm{Zr}$ containing but that some $\mathrm{Sr}$ is still detected throughout the profile. The observation of $\mathrm{Sr}$ and $\mathrm{Zr}$ signal might be related to cation interdiffusion, with the formation of an $\mathrm{SrZrO}_{3}$-related perovskite, as it is regularly observed at the interfaces between Sr-containing perovskite cathodes like LSM and YSZ electrolyte in SOFCs [20]. However, it could not be ruled out that it is related with a particular nanometric arrangement of STO and YSZ domains across the thickness of the sample specimen.

\subsubsection{Multilayer electrical conductance}

AC impedance of the $\mathrm{STO}(10 \mathrm{~nm}) / \mathrm{YSZ}(1 \mathrm{~nm}) / \mathrm{STO}(10 \mathrm{~nm})$ trilayers was measured at temperatures from 100 to $500^{\circ} \mathrm{C}$ in air with a frequency range of $20 \mathrm{~Hz}$ to $2 \mathrm{MHz}$. They generally show three distinct semicircles with associated capacitances in the $\mathrm{pF}, \mathrm{nF}$, and $\mathrm{mF}$ ranges. The higher frequency semicircle (capacitances in $\mathrm{pF}$ range) was attributed to the bulk charge transport in the films, and the temperature dependent DC conductance was then extracted for the different heterostructures. Figure $7(a)$ shows the Arrhenius plot of the conductances measured for a trilayer with a nominal YSZ thickness of $1 \mathrm{~nm}$. For comparison the graph also includes the conductances measured for, (a) a 10nm STO layer on STO(001), (b) a 10nm YSZ layer on $\mathrm{Al}_{2} \mathrm{O}_{3}$ (1-102) deposited in the same conditions of our experiment and (c) the conductance reported for sputtered STO/YSZ(1 nm)/STO trilayers obtained by Garcia- 
Barriocanal et al. [15]. The conductances observed in the STO/YSZ/STO trilayers are much higher than would be expected for bulk STO or YSZ materials with the corresponding nominal thicknesses of $10 \mathrm{~nm}$ and $1 \mathrm{~nm}$, respectively. However, the conductance shown by the single 10nm STO film is also several orders of magnitude higher than expected for pure (nominally undoped) STO material. It is important to point out that the measurement of the conductance of a STO(001) single crystal substrate showed already a conductance larger than expected for undoped STO. It did not significantly differ from that of $10 \mathrm{~nm}$ STO/STO(100) in the high temperature region (not shown in the graph). This could only be related to an electronic ( $p$-type) conductivity induced by the presence of acceptor impurities in STO crystals. It is known that commercial nominally-undoped STO substrates may contain traces of $\mathrm{Mg}, \mathrm{Al}, \mathrm{Mn}$ or $\mathrm{Fe}$ in the ppm range [16]. Whether the deposited 10nm STO film further increases the conductance could not be clearly observed in our experiment, although it can not be ruled out that a slight deviation in the film stoichiometry may have some relevance in the overall charge transport for thicker layers.

It is clear from Figure 7(a) that the STO/YSZ/STO trilayers exhibit a conductance that is greater than the $10 \mathrm{~nm}$ STO layer by about two orders of magnitude which is in agreement with those values previously reported although slightly below conductivity values in ref. [15]. This discrepancy is very likely to be attributed to the different microstructure with poorly connected islands embedded in a STO matrix in our films, rather than continuous YSZ layers. Similar activation energies of about $0.70 \mathrm{eV}$ in both cases give additional support to the fact that the charge transport mechanism might be of the same origin. Janek et al [13-15] predicted a maximum limit in ionic mobility enhancement of about 2.5 orders of magnitude in fully strained (7\%) epitaxial YSZ on STO although they did not take into account possible charge transfer 
effects or complex structure modifications at the interface. Since we did not observe any significant part of the multilayers that was consistent with the presence of fully strained epitaxial YSZ(001)//STO(001) it is very unlikely that this enhancement observed can be uniquely attributed to the YSZ ionic conductivity. Either the incorporation of substitutional $\mathrm{Y}$ and $\mathrm{Zr}$ below the detection limit of EELS in the surrounding STO matrix, or, the formation of an interlayer in the region where YSZ was expected to appear, possibly Y-doped $\mathrm{Sr}(\mathrm{Ti}, \mathrm{Zr}) \mathrm{O}_{3}$ perovskite, is a more probable explanation for conductivity observed in the films as opposed to an enhancement of YSZ ionic conductivity. The observation of the brighter Z-contrast areas around the YSZ islands and the chains in Fig. 5 would support this hypothesis. This might be in agreement with the observations made on Y-doped $\mathrm{SrZrO}_{3}$ single crystals [21], $\mathrm{SrZr}_{0.95} \mathrm{Y}_{0.05} \mathrm{O}_{3} / \mathrm{STO}$ thin film heterostructures [22], and Fe-doped STO crystals [23] which have shown dominant $p$-type electronic conductivities in the high $p \mathrm{O}_{2}$ region with enhanced values of about $10^{-4} \mathrm{~S} / \mathrm{cm}$ at $450{ }^{\circ} \mathrm{C}$ in comparison to their parent undoped compounds, with a $+1 / 4 \mathrm{pO}_{2}$ power dependence in all cases under oxidising conditions (in dry $\mathrm{O}_{2}$ ). The reported activation energies from $0.67 \mathrm{eV}$ (at lower temperatures) to $0.84 \mathrm{eV}$ (at high temperature) [21] are also comparable with our data.

\subsubsection{Oxygen partial pressure dependence}

In order to determine the electronic or ionic nature of the observed conductivities we analysed the conductance of a trilayer film with $1 \mathrm{~nm}$ YSZ nominal thickness at different $p \mathrm{O}_{2}$ from $10^{-4}$ to 1 bar. The experiment was performed at a constant temperature of $450{ }^{\circ} \mathrm{C}$ after allowing enough time to reach stationary conditions (from $30 \mathrm{~min}$ to $1 \mathrm{hr}$ ) to ensure that all parts of the heterostructure have reached equilibrium and the experiment is not hampered by possible slow kinetics in the transverse oxygen diffusion induced by the layered structure. Figure $7(b)$ 
shows the corresponding impedance plots, along with the $p \mathrm{O}_{2}$ dependence of the high frequency semicircle conductance in Fig. 7(c). The graph shows a power law with an exponent $0.21( \pm$ $0.02)$ which is very close to the value of 0.195 reported for the bulk transport in Fe-doped $\left(10^{8}\right.$ $\mathrm{cm}^{-3}$ ) $\mathrm{SrTiO}_{3}$ bicrystals [24], as well as to other acceptor-doped STO [16,23] and Y-doped $\mathrm{SrZrO}_{3}$ [23], and is considered as characteristic for electronic (hole) $p$-type conductivity in those cases where oxygen vacancies are equilibrated by a constant concentration of acceptor impurities, although from this measurements is still difficult to figure out the nature of the dopants.

\subsubsection{Oxygen isotope exchange depth profiling}

To further ascertain the nature of the charge carriers ${ }^{18} \mathrm{O}$ IEDP experiments were carried out followed by analysis of the films using SIMS. In a first set of experiments samples with a clean cleaved lateral surface, or with a trench, were exchanged at two different temperatures (450 and $500{ }^{\circ} \mathrm{C}$ ) for 2 hours. Figure 8 shows the depth profile of the background corrected ${ }^{18} \mathrm{O}$ fraction normalised to the gas concentration, $\mathrm{C}^{\prime}(\mathrm{x})$, obtained from the central area of a crater, approximately $400 \times 450 \mu \mathrm{m}^{2}$, from a [STO/YSZ]x10 multilayer film exchanged at $450{ }^{\circ} \mathrm{C}$. The value of the depth has been calculated by applying a correction which takes into account the different sputtering rates observed as the SIMS primary ion beam goes through the different layers (STO capping layer, YSZ/STO multilayer and STO substrate). The crater was sputtered at a lateral distance of approximately $1 \mathrm{~mm}$ from both the open trench and from the side of the sample, i.e. at a distance much greater than the diffusion length for bulk YSZ given the conditions of the anneal. It can be seen that the ${ }^{18} \mathrm{O}$ fraction on the STO surface is 0.375 , decreasing to a value of 0.012 through the first $135 \mathrm{~nm}$ of STO capping layer and shows a 
shallow diffusion profile in the capping layer. Following this the ${ }^{18} \mathrm{O}$ fraction abruptly decreases to a value of zero at a depth of $\sim 144 \mathrm{~nm}$ in a depth interval of less than $10 \mathrm{~nm}$. Throughout the multilayer [STO/YSZ] structure the isotopic fraction is constant and essentially zero, within experimental error. In the inset in Fig. 8 the secondary ion signals for ${ }^{18} \mathrm{O}^{-},{ }^{16} \mathrm{O}^{-},{ }^{89} \mathrm{Y}^{16} \mathrm{O}^{-}$and ${ }^{90} \mathrm{Zr}^{16} \mathrm{O}^{-}$measured for the same crater are shown. By following the number of counts associated with the different elements we can identify the outermost layer of the multilayer structure (i.e. beneath the cap) as the signals corresponding to ${ }^{89} \mathrm{Y}^{16} \mathrm{O}^{-}$and ${ }^{90} \mathrm{Zr}^{16} \mathrm{O}^{-}$abruptly increase. Moreover, each of the 10 YSZ layers can be clearly distinguished, corresponding to the 10 maxima for both the ${ }^{89} \mathrm{Y}^{16} \mathrm{O}^{-}$and ${ }^{90} \mathrm{Zr}^{16} \mathrm{O}^{-}$signals. The width of each of these maxima is 2.8-3.2 $\mathrm{nm}$, which is roughly the same thickness value observed for the YSZ islands identified by HRTEM. Close inspection of the depth profile at a depth of $135 \mathrm{~nm}$ reveals that the ${ }^{18} \mathrm{O}$ fraction decreases to zero before the first YSZ layer is reached. It seems that the multilayer structure is, rather surprisingly, acting as a barrier to oxygen diffusion in the direction perpendicular to the layers. The observation that there is no discernable ${ }^{18} \mathrm{O}$ fraction in the multilayer structure also means that no isotope has been able to diffuse along the layer structure into the main body of the sample which would have been the case if the diffusion of oxygen had been enhanced by several orders of magnitude. On this same sample, another crater was sputtered through an area which included the open trench. Once the STO capping layer had been sputtered off and the multilayer had been reached, longitudinal concentration profiles were measured from the open trench towards the centre of the sample. No lateral oxygen diffusion was found to occur from the open sides into the multilayer STO/YSZ layers. Similar findings were observed for the [STO/YSZ]X10 multilayers and STO/YSZ/STO trilayer films exchanged at a higher temperature of $500{ }^{\circ} \mathrm{C}$, and for the samples exchanged at $450{ }^{\circ} \mathrm{C}$ which had the exposed surface covered with an Ag layer to enhance the surface exchange. 


\section{Conclusions}

We have demonstrated that the combination of YSZ and STO material in the form of thin epitaxial films induces an enhancement of the electrical conductivity above those values observed for STO and YSZ films deposited under the same conditions. Together, the power dependence of the electrical conductivity upon oxygen partial pressure variations with exponent $+1 / 4$ and the observation of a lack of ${ }^{18} \mathrm{O}$ diffusion along the interfaces are unequivocal proof that the main contribution to the conductance in these films is related to electronic conductivity rather than ionic. The particular microstructure of our films which shows interfaces between YSZ and STO with different orientations rules out the possibility that the effect is related to a particular interface orientation. It is not proven that a microstructure with a larger degree of connectivity between YSZ domains than in our films, which consist mainly of isolated YSZ islands embedded in the epitaxial STO matrix, would induce a certain degree of ionic diffusivity. However, the absolute value of the electronic conductance attained in our films, along with the apparent activation energies, are very similar to those reported in previous work by GarciaBarriocanal et al [15]. The origin of the conductivity enhancement seems to be related to the cation interdiffusion generated at the interfaces, and very likely to the formation of a dopedstrontium zirconate perovskite as is suggested from the EELS analysis, and the values of activation energies and $\mathrm{pO}_{2}$ dependence reported for our films. Given the electronic nature of these nanocomposite YSZ/STO films it is not possible to use them as an electrolyte in solid oxide fuel cells. However, if the connectivity between YSZ domains is enhanced and the ionic conductance reaches at least the values corresponding to bulk YSZ, these films could find a 
technological application as cathode material in ultrathin monolithic epitaxial SOFC devices, provided the cathode surface shows enhanced catalytic effect for oxygen reduction.

\section{Acknowledgements}

The authors would like to acknowledge Richard J. Chater for his help with SIMS analysis. The authors also acknowledge the financial support of Spanish Government through projects: MAT2008-04931 and Consolider-Ingenio 2010-CSD2008-023. Two of the authors (A.C. and M.B.) acknowledge the support of their postdoctoral fellowships from the Spanish Ministry of

Science and Innovation. DWM acknowledges the support of the Royal Academy of Engineering. 


\section{References}

[1] B.C.H. Steele, A. Heinzel, Nature 414 (2001) 345.

[2] N.P. Brandon, S. Skinner, B.C.H. Steele, Annu. Rev. Mater. Res. 33 (2003) 183.

[3] J. Maier, Phys Chem. Chem. Phys. 11 (2009) 3011.

[4] X.X. Guo, J. Maier, Adv. Mater. 21 (2009) 2619.

[5] I. Kosacki, C.M. Rouleau, P.F. Becher, J. Bentley, D.H. Lowndes, Solid State Ionics 176 (2005) 1319.

[6] Y.-M. Chiang, E.B. Lavik, I. Kosacki, H.L. Tuller, J.Y. Ying, Appl. Phys. Lett. 69 (1996) 185 .

[7] N. Sata, K. Eberman, K. Eberl, J. Maier, Nature 408 (2000) 946.

[8] X.X. Guo, J. Maier, Adv. Funct. Mater. 19 (2009) 96.

[9] S. Azad, O.A. Marina, C.M. Wang, L. Saraf, V. Shutthanandan, D.E. McCready, A. ElAzab, J.E. Jaffe, M.H. Engelhard, C.H.F. Peden, S. Thevuthasan, Appl. Phys. Lett. 86 (2005) 131906.

[10] I. Kosacki, invention disclosure \#5-153.1685, February 2006; reported in workshop: Accelerated Materials Discovery for Energy Storage and Conversion Devices, St Antony’s College, Oxford, UK, 2nd - 4th April 2007, Ref: UKERC/MR/MP/2007/012, www.ukerc.ac.uk.

[11] A. Peters, C. Korte, D. Hesse, N. Zakharov, J. Janek, Solid State Ionics 178 (2007) 67.

[12] C. Korte, A. Peters, J. Janek, D. Hesse, N. Zakharov, Phys. Chem. Chem. Phys. 10 (2008) 4623.

[13] C. Korte, N. Schichtel, D. Hesse, J. Janek, Chem. Monthly 140 (2009) 1069.

[14] N. Schichtel, C. Korte, D. Hesse, J. Janek, Phys. Chem. Chem. Phys., 11 (2009) 3043. 
[15] J. Garcia-Barriocanal, A. Rivera-Calzada, M. Varela, Z. Sefrioui, E. Iborra, C. Leon, S.J. Pennycook, J. Santamaria, Science 321 (2008) 676.

[16] X. Guo, Comment on "Colossal ionic conductivity at interfaces of epitaxial $\mathrm{ZrO}_{2}: \mathrm{Y}_{2} \mathrm{O}_{3} / \mathrm{SrTiO}_{3}$ heterostructures", Science 324 (2009) 465a; plus Supporting Online Material at www.sciencemag.org/cgi/content/full/324/5926/465a/DC1.

[17] J. Garcia-Barriocanal, A. Rivera-Calzada, M. Varela, Z. Sefrioui, E. Iborra, C. Leon, S.J. Pennycook, J. Santamaria, Response to comment on "Colossal ionic conductivity at interfaces of epitaxial $\mathrm{ZrO}_{2}: \mathrm{Y}_{2} \mathrm{O}_{3} / \mathrm{SrTiO}_{3}$ heterostructures" Science 324 (2009) 465b.

[18] R. Bachelet, F. Sanchez, J. Santiso, C. Munuera, C. Ocal, J. Fontcuberta, Chem. Mater. 21 (2009) 2494.

[19] M. Leonhardt, R. A. De Souza, J. Claus, J. Maier, J. Electrochem. Soc. 149 (2002) J19.

[20] G.Ch. Kostogloudis, G. Tsiniarakis, Ch. Ftikos, Solid State Ionics 135 (2000) 529.

[21] J. Müller, K.D. Kreuer, J. Maier, S. Matsuo, M. Ishigame, Solid State Ionics 97 (1997) 421.

[22] N. Kuwata, N. Sata, T. Tsurui, H. Yugami, Jpn. J. Appl. Phys. 44 (2005) 8613.

[23] K. Sasaki, J. Claus and J. Maier, Solid State Ionics 121 (1999) 51.

[24] Z. Zhang, W. Sigle, R. A. De Souza, W. Kurtz, J. Maier, M. Rühle, Acta Materialia 53 (2005) 5007. 


\section{Figure Captions:}

Fig. 1. RHEED pattern (left) obtained for the homoepitaxial growth of $\mathrm{SrTiO}_{3}$ onto a $\mathrm{SrTiO}_{3}(001)$ substrate along the [100] zone axis. The curve on the right corresponds to the variation of the integrated intensity of the main reflected beam (bright spot in the centre of the RHEED image) during the first 60 pulses corresponding to the growth of about 12 monolayers $(4.7 \mathrm{~nm})$. The inset shows a zoom of the first 16 pulses ( 3 monolayers) where the recovery after each individual pulse $(1 \mathrm{sec})$ is clearly observed $(t=0 \mathrm{sec}$ corresponds to the first laser shot).

Fig. 2. AFM images $\left(1 \mathrm{x} 1 \mu \mathrm{m}^{2}\right)$ of the top surface morphology of $\mathrm{STO}(10 \mathrm{~nm}) / \mathrm{YSZ} /$ STO(10 nm) trilayers deposited by PLD on STO(001) single crystal substrates with different nominal thickness of the YSZ interlayer (left); and corresponding RHEED patterns of the top STO layer along the [100] zone axis (right). The image shows also rms roughness values of the films.

Fig. 3. RHEED experimental pattern of the YSZ surface of a $\operatorname{STO}(10 \mathrm{~nm}) / \mathrm{YSZ}(5 \mathrm{~nm})$ bilayer deposited on $\mathrm{SrTiO}_{3}(001)$ substrate. The crystal was oriented along the [100]STO zone axis. The red dots correspond to a simulated pattern of the combination of two $15^{\circ}-$ tilted (111)YSZ domains $(a)$; scheme of the relative orientation of YSZ and STO domains (b); and XRD pole figures of 111 and 200 YSZ reflections measured at fixed $2 \theta$ angles of 30.0 and $34.8^{\circ}$, respectively, for the same STO/YSZ bilayer $(c)$. 
Fig. 4. AFM image $\left(0.5 \times 0.5 \mu \mathrm{m}^{2}\right)$ of the surface of a $[\operatorname{STO}(10 \mathrm{~nm}) / \mathrm{YSZ}(1 \mathrm{~nm})] \times 10$ multilayer grown on a $\mathrm{SrTiO}_{3}(001)$ substrate $(a)$. High resolution XRD pattern of the 002 Bragg reflection of the same STO/YSZ multilayer $(b)$. The satellite peaks correspond to a multilayer periodicity of $9.6 \mathrm{~nm}$. Corresponding X-ray reflectivity curve showing measured values for the multilayer $(c)$. Both XRD graphs show also simulated curves (in grey) for equivalent STO/YSZ multilayers with perfectly flat interfaces.

Fig. 5. Cross section TEM images (in HAADF mode) of trilayer STO(10 nm) / YSZ(1 nm) / STO $(10 \mathrm{~nm})$ heterostructure $(a)$ and detail of the YSZ islands with different crystal orientation $(b)$; and $[\mathrm{STO}(10 \mathrm{~nm}) / \mathrm{YSZ}(1 \mathrm{~nm})] \times 10$ multilayer $(c)$, along with details of the different observed microstructures consisting of YSZ islands in consecutive levels ( $d$ and $e$ ), and Y, Zr-containing "chain-like" structure $(f)$. Note that surronding STO structure is perfectly coherent throughout the film.

Fig. 6. EELS analysis of the film in the areas indicated in the cross section image $(a)$. Typical wide EELS spectra of pure YSZ and STO materials, obtained in the central area of \#1 and \#2 scans, respectively, showing selected energy windows for $\mathrm{Sr}_{3}$ and $\mathrm{Zr} \mathrm{L}_{3}$ lines (b); along with normalised HAADF and EELS intensity profiles obtained along \#1, \#2 and \#3 scans $(c)$. 
Fig. 7. Arrhenius plot of the planar AC conductance measured for the different heterostructures in air: trilayer STO/YSZ(1nm)/STO (blue cicles), $10 \mathrm{~nm}$ STO film (black triangles), $10 \mathrm{~nm}$ YSZ film (black squares), as well as previously reported values for a $\mathrm{STO} / \mathrm{YSZ}(1 \mathrm{~nm}) / \mathrm{STO}$ trilayer in ref [15] for comparison (red circles) (a); and partial oxygen pressure dependence of the impedance plot of the high frequency semicircle at 450 ${ }^{\circ} \mathrm{C}$ for the STO/YSZ(1 nm)/YSZ trilayer, from 1 bar pure oxygen down to $3 \times 10^{-4}$ bar $(b)$, along with corresponding conductances showing a power law dependence with exponent $0.21( \pm 0.02)(c)$.

Fig. 8. Isotopic ${ }^{18} \mathrm{O} /$ background corrected fraction in a depth profile measured by SIMS on a $[\mathrm{STO}(10 \mathrm{~nm}) / \mathrm{YSZ}(1 \mathrm{~nm})] \times 10$ multilayer capped with $135 \mathrm{~nm}$ STO laterally exposed to a ${ }^{18} \mathrm{O}$-enriched atmosphere at $450{ }^{\circ} \mathrm{C}$ through an opening in the film. The inset shows the intensity signal measured for ${ }^{18} \mathrm{O},{ }^{16} \mathrm{O},{ }^{89} \mathrm{Y}^{16} \mathrm{O}^{-}$and ${ }^{90} \mathrm{Zr}^{16} \mathrm{O}^{-}$ions. 

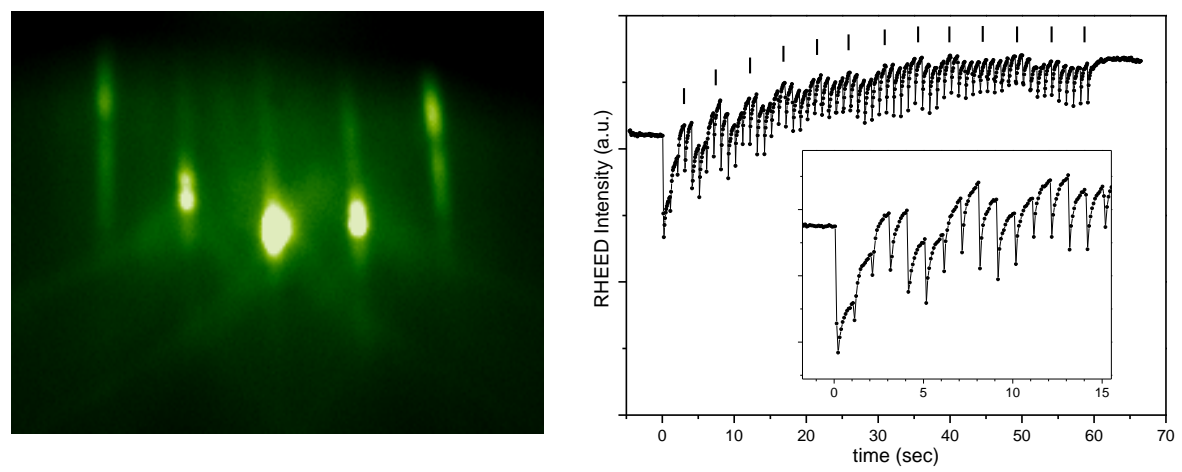

Figure 1 


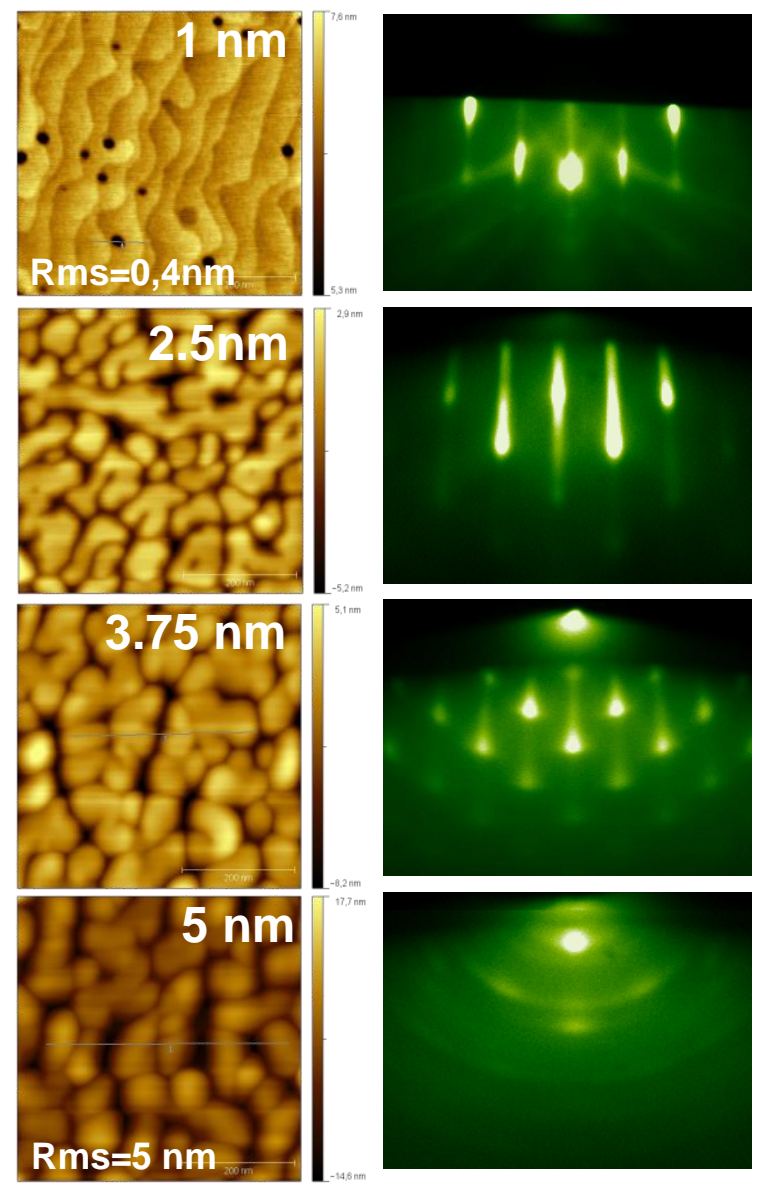

Figure 2 


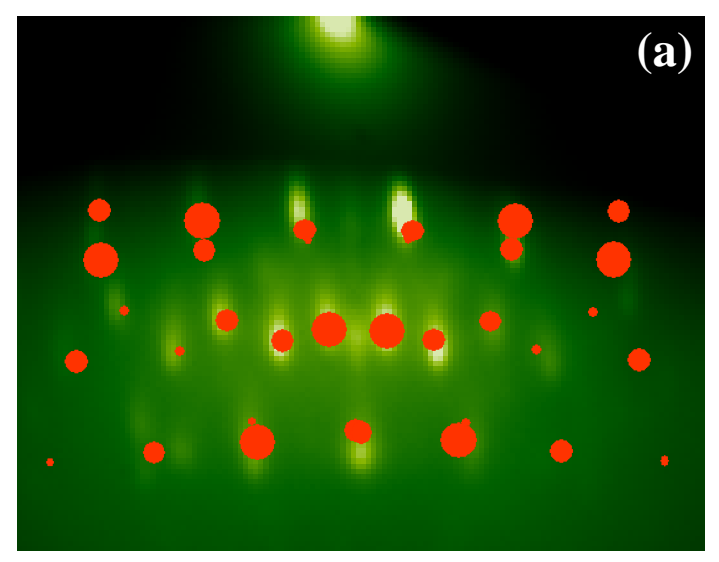

(b)

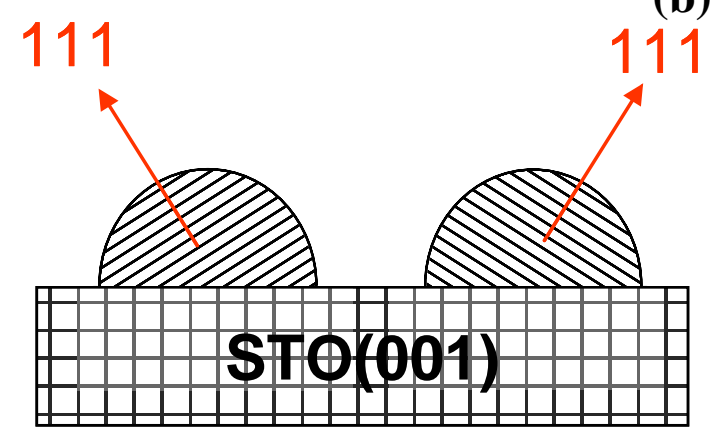

(c)

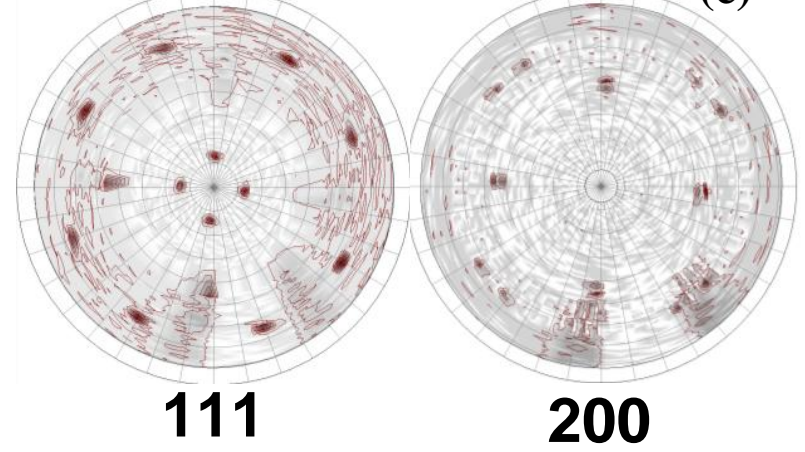

Figure 3 

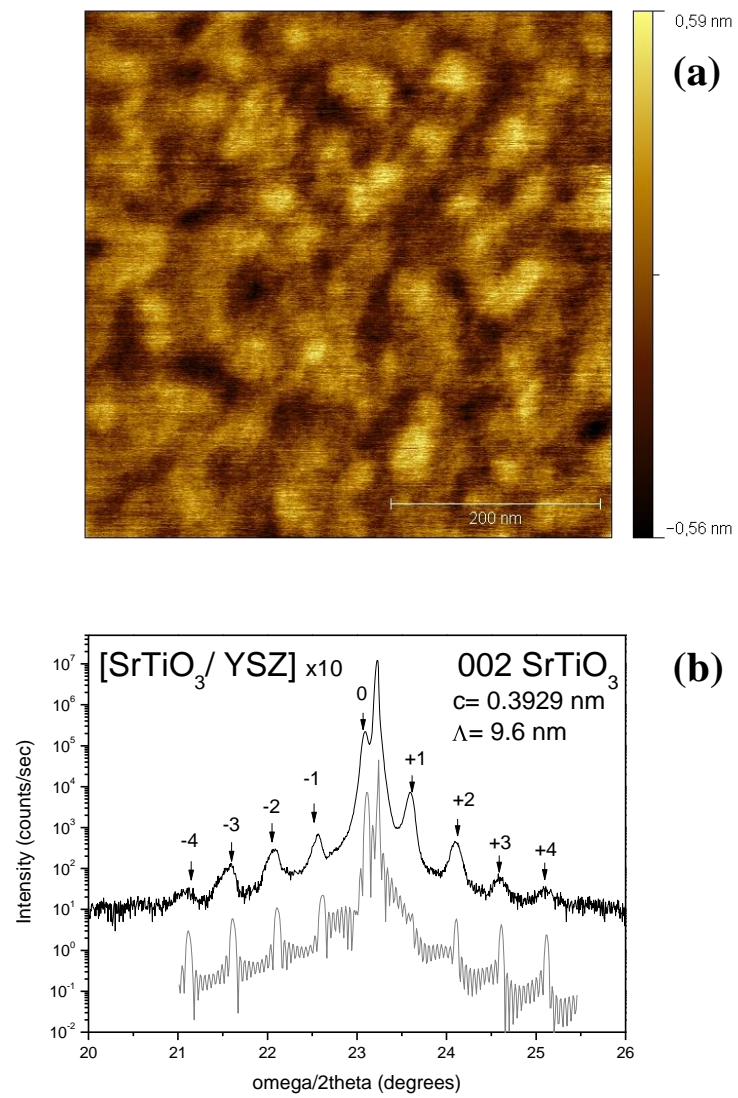

(b)

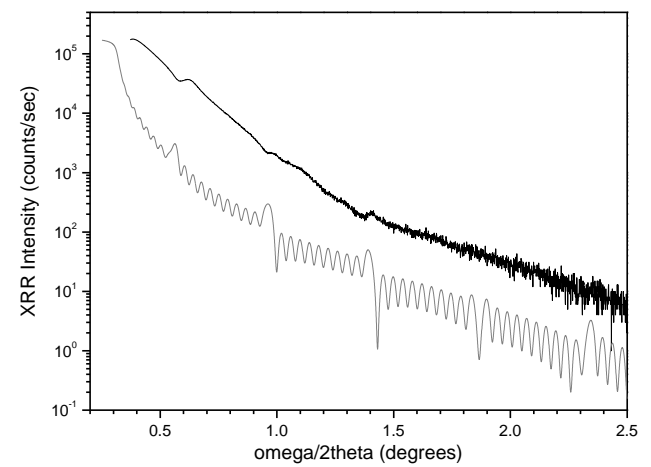

(c)

Figure 4 


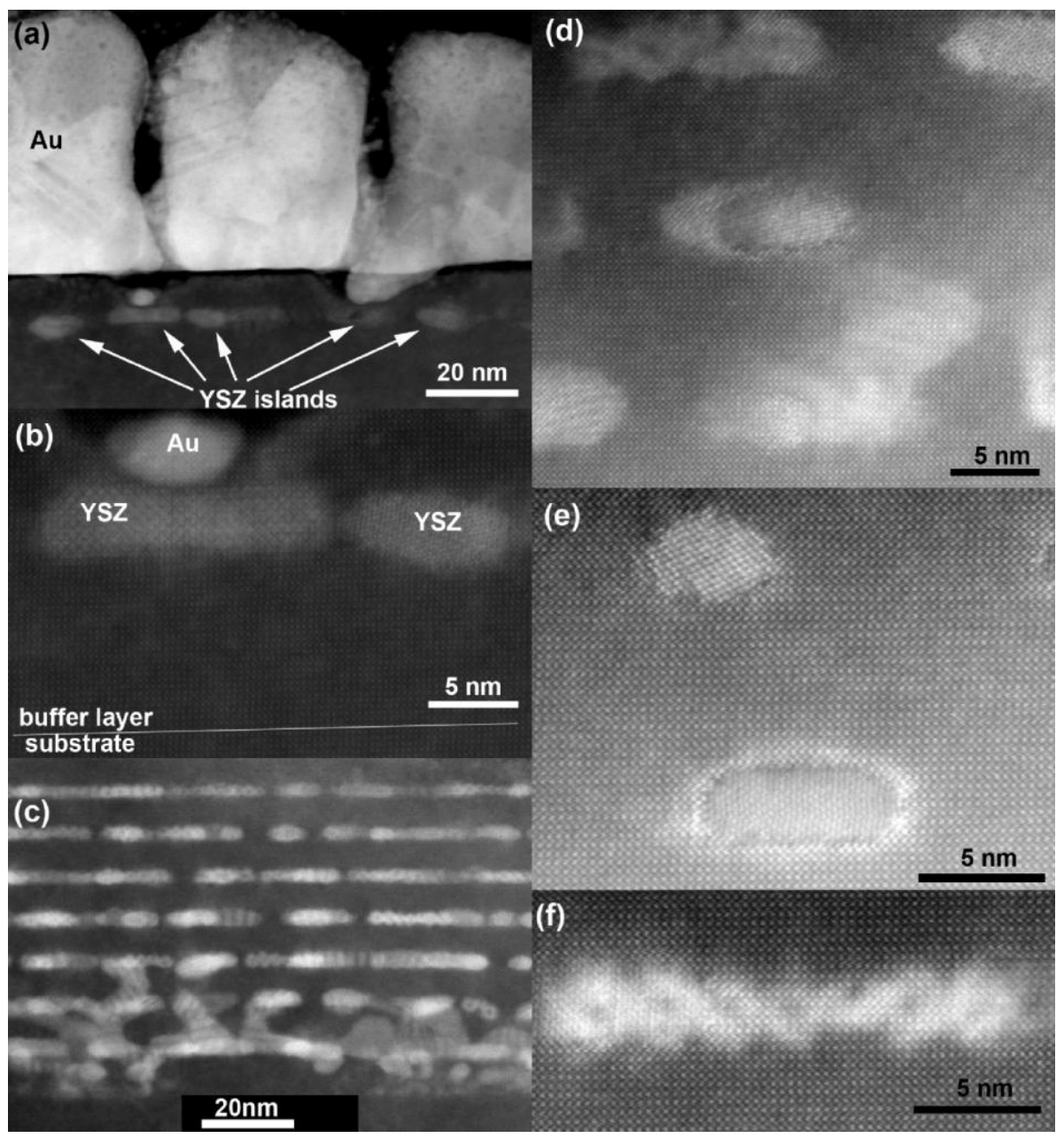

Figure 5 


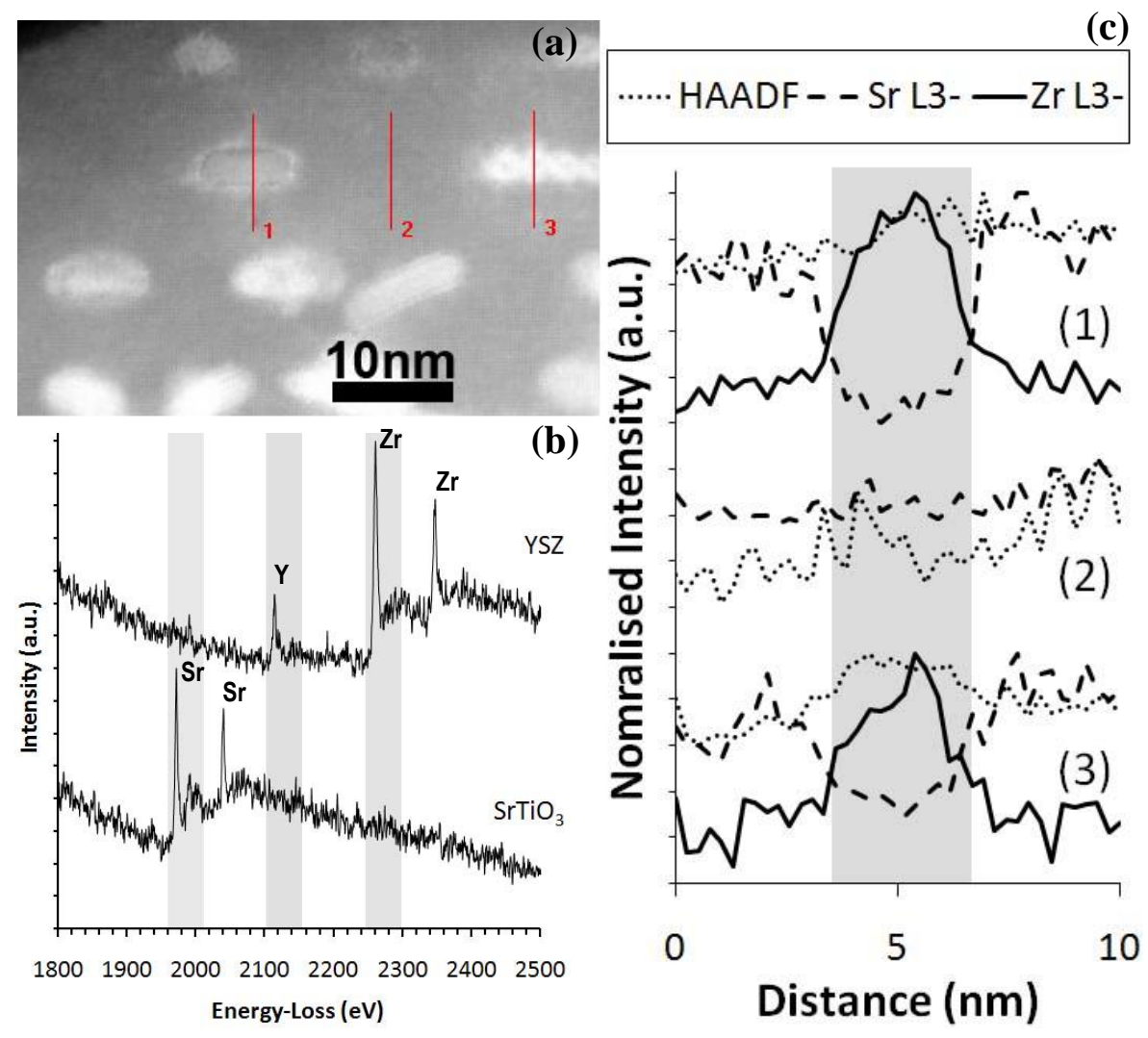

Figure 6 

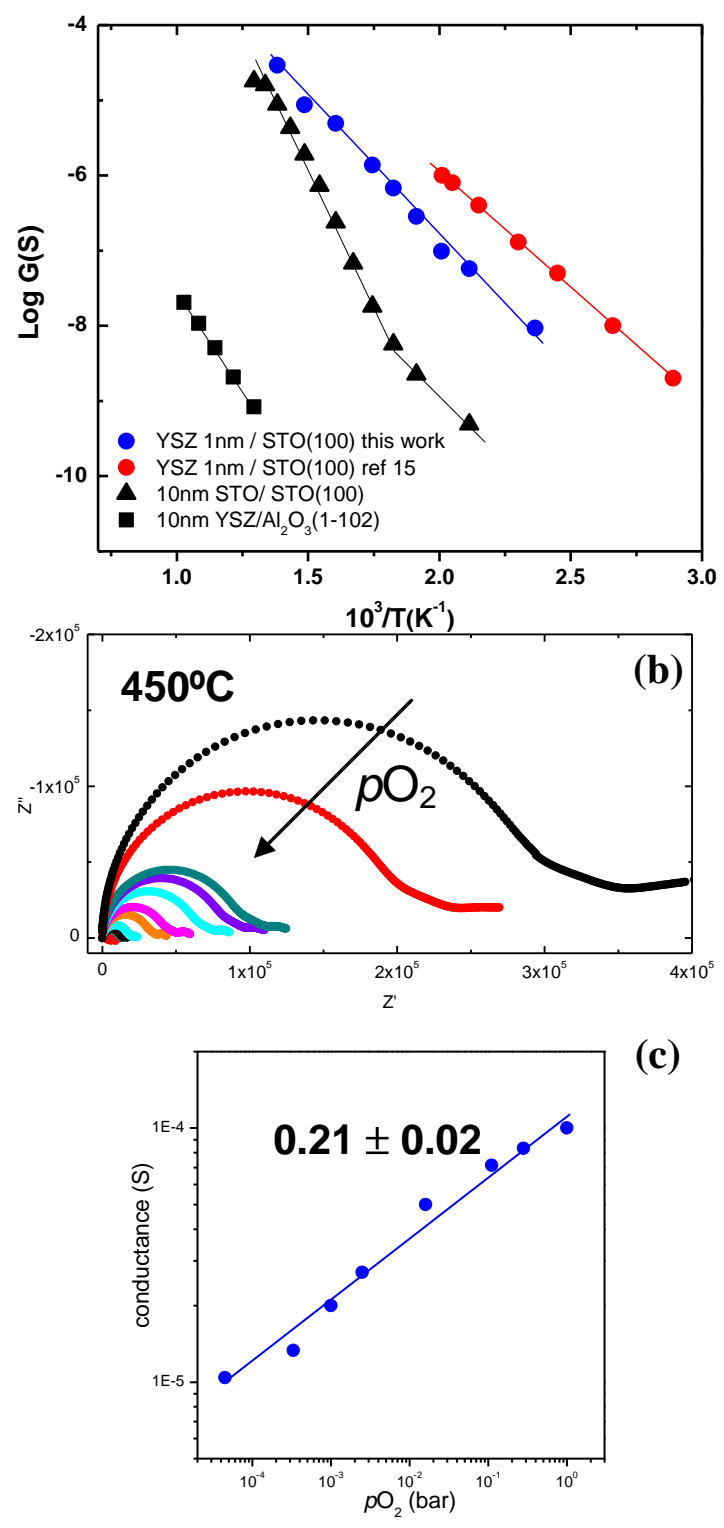

(c)

Figure 7 


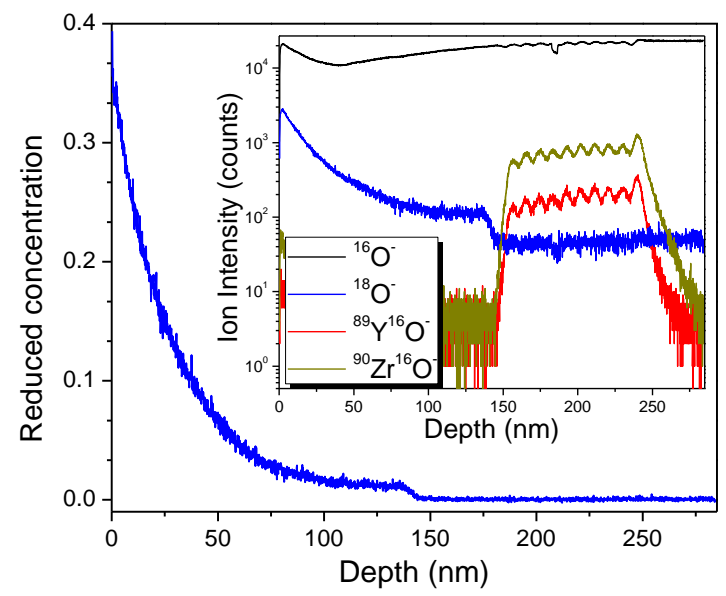

Figure 8 